\section{Lingering Binocular Vision Issues after a Suspected Concussion: A Case Study}

\section{Abstract}

This case study examines long-term vision related issues after a suspected concussion. After assessments, it became apparent that the vision issues were widespread and ongoing vision therapy was conducted. A 12-month follow up revealed significant improvement in all oculomotor metrics, although the patient remained outside normative ranges.

Keywords: Binocular vision; Concussion; Eye tracking; Vision

\section{Melissa Hunfalvay \\ Department of Science, Right Eye, LLC, 7979 Old Georgetown Road, 8th Floor, Bethesda, MD 20814, USA}

Corresponding author: Melissa Hunfalvay

” melissa@righteye.com

Chief Science Officer, Department of Science, Right Eye, 7979 Old Georgetown Road, 8th Floor, Bethesda, MD 20814, USA.

Tel: 240-357-0026

Citation: Hunfalvay M. Lingering Binocular Vision Issues after a Suspected Concussion: A Case Study. Transl Biomed. 2017, 8:2.

Figure 1. Typical gaze trail patterns, of age-related comparison, are shown in Figure 2.

Compared to normative data ranges the patient fell outside the norms on all oculomotor metrics (Table 1). Saccade: fast

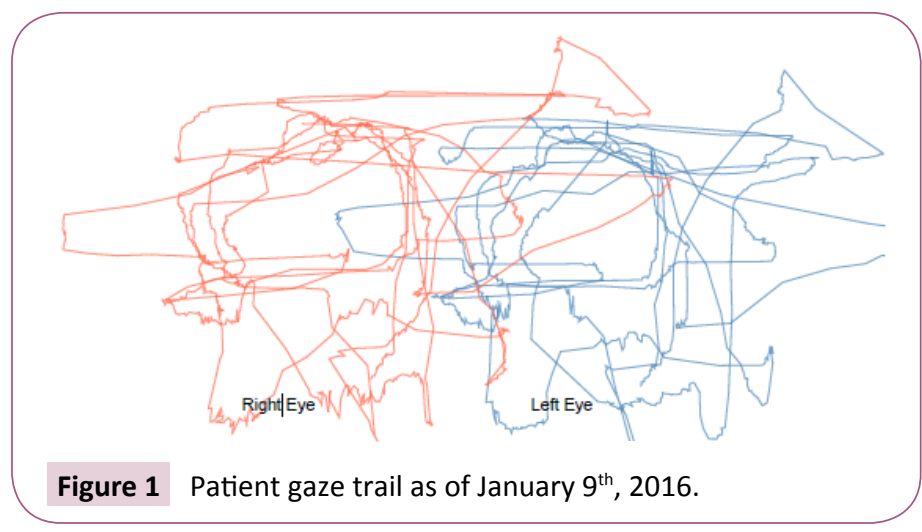

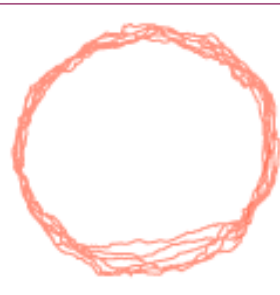

Right Eye

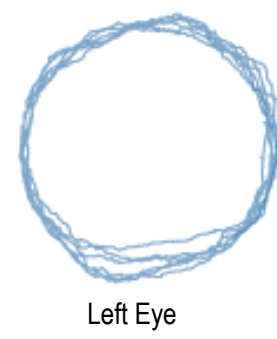

Left Eye
Figure 2 Typical age-related gaze trail. oculomotor behavior. This is a digitized, eye-tracked, form of the VOMS. The gaze trail of her eye movements can be seen in 
eye movement. Fixation: stopping point. Smooth pursuit: eye follows target. Velocity error: error in judgement of speed. Horizontal synchronization: judgement in relation to target and $X$-coordinate. Vertical synchronization: judgement in relation to target and Y-coordinate. Predictive: eye in-front of target. Latent: eye behind target. On January $6^{\text {th }}, 2017$ patient was again tested using RightEye (Figure $\mathbf{3}$ and Table 2). Testing showed marked improved since 2016 with all metrics moving in the direction of population norms. The predictive smooth pursuit metric increased toward population norms; however a lower number is preferred.

Table 3 shows the percentage of improvement over the 12 months between Right Eye assessments. Green shows the improved metrics in the desired direction.

\section{Discussion}

Results of this case study show ongoing oculomotor deficits compared to age-related population norms after a suspected concussion. Extensive vision therapy, improved results however, the patient continues to remain outside normal ranges.

\section{Conclusion}

This case study highlights the possible long-term effects of vision issues after a concussion, along with a quantitative method for ongoing clinical analysis.

Table 1 Patients eye movement metrics as of January $9^{\text {th }}, 2016$.

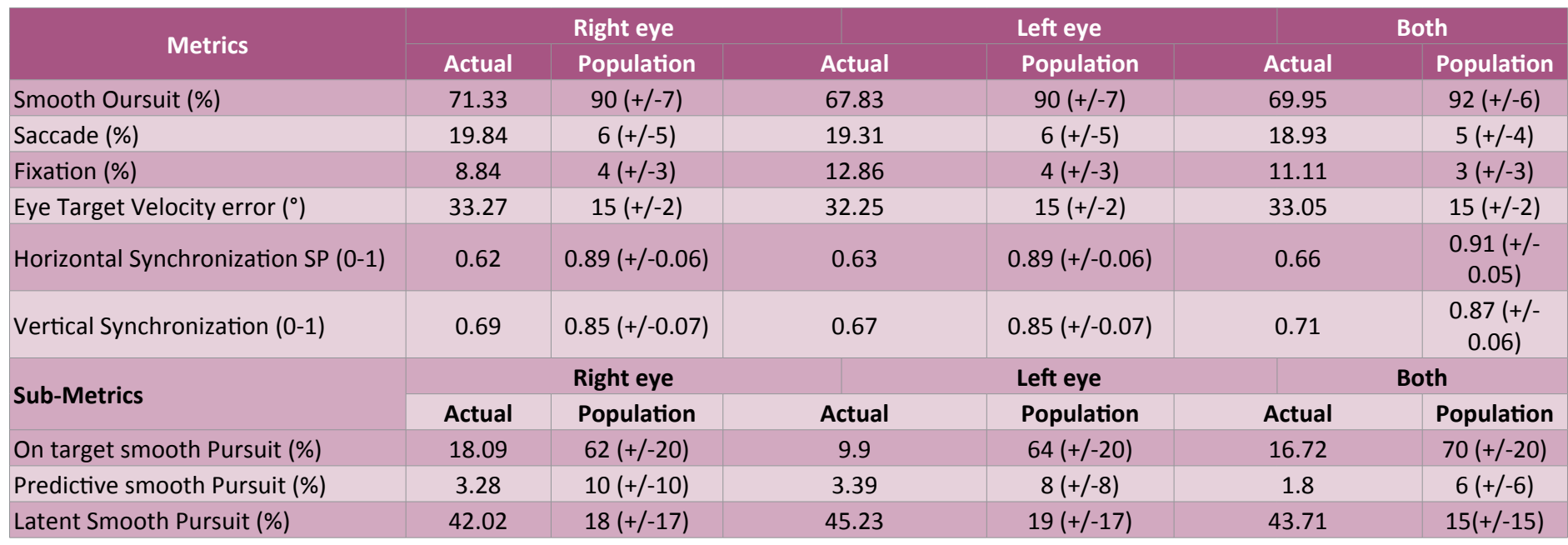

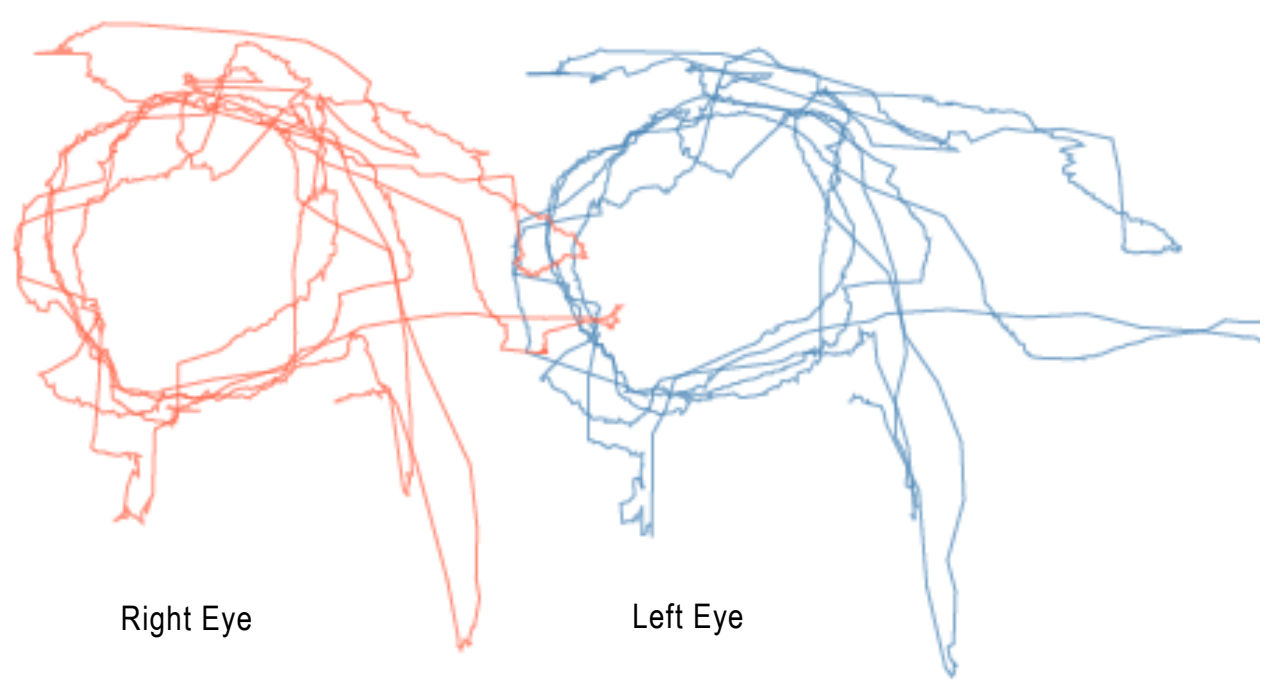

Figure 3 Patient gaze trail as of January $6^{\text {th }}, 2017$. 
Table 2 Patients eye movement metrics as of January $6^{\text {th }}, 2017$.

\begin{tabular}{|c|c|c|c|c|c|c|}
\hline \multirow{2}{*}{ Metrics } & \multicolumn{2}{|c|}{ Right eye } & \multicolumn{2}{|c|}{ Left eye } & \multicolumn{2}{|c|}{ Both } \\
\hline & Actual & Population & Actual & Population & Actual & Population \\
\hline Smooth Oursuit (\%) & 76.53 & $90(+/-7)$ & 79.38 & $90(+/-7)$ & 78.22 & $92(+/-6)$ \\
\hline Saccade (\%) & 12.95 & $6(+/-5)$ & 13.11 & $6(+/-5)$ & 12.9 & $5(+/-4)$ \\
\hline Fixation (\%) & 10.52 & $4(+/-3)$ & 7.51 & $4(+/-3)$ & 8.89 & $3(+/-3)$ \\
\hline Eye Target Velocity error $\left({ }^{\circ}\right)$ & 21.36 & $15(+/-2)$ & 22.08 & $15(+/-2)$ & 21.82 & $15(+/-2)$ \\
\hline Horizontal Synchronization SP (0-1) & 0.81 & $0.89(+/-0.06)$ & 0.8 & $0.89(+/-0.06)$ & 0.81 & $0.91(+/-0.05)$ \\
\hline Vertical Synchronization (0-1) & 0.79 & $0.85(+/-0.07)$ & 0.73 & $0.85(+/-0.07)$ & 0.76 & $0.87(+/-0.06)$ \\
\hline \multirow{2}{*}{ Sub-Metrics } & \multicolumn{2}{|c|}{ Right eye } & \multicolumn{2}{|c|}{ Left eye } & \multicolumn{2}{|c|}{ Both } \\
\hline & Actual & Population & Actual & Population & Actual & Population \\
\hline On target smooth Pursuit (\%) & 47.39 & $62(+/-20)$ & 45.37 & $64(+/-20)$ & 47.44 & $70(+/-20)$ \\
\hline Predictive smooth Pursuit (\%) & 11.26 & $10(+/-10)$ & 10.73 & $8(+/-8)$ & 10.26 & $6(+/-6)$ \\
\hline Latent Smooth Pursuit (\%) & 12.7 & $18(+/-17)$ & 16.46 & $19(+/-17)$ & 14.12 & $15(+/-15)$ \\
\hline
\end{tabular}

Table 3 Percentage of improvement over the 12 months between Right eye assessments.

\begin{tabular}{|c|c|c|c|c|c|c|}
\hline \multirow{2}{*}{ Metrics } & \multicolumn{2}{|c|}{ Right eye } & \multicolumn{2}{|c|}{ Left eye } & \multicolumn{2}{|c|}{ Both } \\
\hline & Actual & Population & Actual & Population & Actual & Population \\
\hline Smooth Oursuit (\%) & $\uparrow 5.2$ & $90(+/-7)$ & 个 11.55 & $90(+/-7)$ & 个 8.27 & $92(+/-6)$ \\
\hline Saccade (\%) & 个 6.89 & $6(+/-5)$ & 个 6.2 & $6(+/-5)$ & 个 6.03 & $5(+/-4)$ \\
\hline Fixation (\%) & 个 4.11 & $4(+/-3)$ & 个 5.35 & $4(+/-3)$ & 个 2.22 & $3(+/-3)$ \\
\hline Eye Target Velocity error $\left({ }^{\circ}\right)$ & 个 11.91 & $15(+/-2)$ & 个 10.17 & $15(+/-2)$ & 个 11.23 & $15(+/-2)$ \\
\hline Horizontal Synchronization SP (0-1) & $\uparrow 0.19$ & $0.89(+/-0.06)$ & 个 0.17 & $0.89(+/-0.06)$ & 个 0.15 & $0.91(+/-0.05)$ \\
\hline Vertical Synchronization (0-1) & $\uparrow 0.1$ & $0.85(+/-0.07)$ & $\uparrow 0.06$ & $0.85(+/-0.07)$ & 个 0.05 & $0.87(+/-0.06)$ \\
\hline \multirow{2}{*}{ Sub-Metrics } & \multicolumn{2}{|c|}{ Right eye } & \multicolumn{2}{|c|}{ Left eye } & \multicolumn{2}{|c|}{ Both } \\
\hline & Actual & Population & Actual & Population & Actual & Population \\
\hline On target smooth Pursuit (\%) & 个 29.3 & $62(+/-20)$ & 个 35.47 & $64(+/-20)$ & 个 30.72 & $70(+/-20)$ \\
\hline Predictive smooth Pursuit (\%) & 个 7.98 & $10(+/-10)$ & 个 7.34 & $8(+/-10)$ & 个 8.46 & $6(+/-6)$ \\
\hline Latent Smooth Pursuit (\%) & 个 29.32 & $18(+/-17)$ & 个 28.77 & $19(+/-17)$ & 个 29.59 & $15(+/-15)$ \\
\hline
\end{tabular}

Note: Green shows the improved metrics in the desired direction. 


\section{References}

1 Contreras R, Kolster R, Voss HU, Ghajar J, Suh M, et al. (2008) Eyetarget synchronization in mild traumatic brain-injured patients. J Biol Phys 34: 381-392.

2 Leigh RJ, Zee DS (2006) The neurology of eye movements. Oxford University Press, UK. 3-16.
3 Maruta J, Suh M, Niogi SN, Mukherjee P, Ghajar J (2010) Visual tracking synchronization as a metric for concussion screening. J Head Trauma Rehabil 25: 293-305.

4 Heitger MH, Anderson TJ, Jones RD, Dalrymple-Alford JC, Frampton $\mathrm{CM}$, et al. (2004) Eye movement and visuomotor arm movement deficits following mild closed head injury. Brain 127: 575-590. 